\title{
Correspondence-Free Alignment of 3D Object Models
}

\author{
Ceyhun Burak Akgül ${ }^{\mathrm{a}}$, Bülent Sankur ${ }^{\mathrm{b}}$, Yücel Yemez ${ }^{\mathrm{b}}$ \\ ${ }^{a}$ Boğaziçi University Electrical -Electronics Engineering Dept., Bebek, Istanbul \\ ${ }^{\mathrm{b}}$ Koç University Computer Engineering Dept., Sarıyer, Istanbul
}

\begin{abstract}
In this work, we present a pose-invariant shape matching methodology for complete 3D object models. Our approach is based on first describing the objects with shape descriptors and then minimizing the distance between descriptors over an appropriate set of geometric transformations. Our chosen shape description methodology is the density-based framework (DBF), which is experimentally shown to be very effective in 3D object retrieval [1]. In our earlier work, we showed that density-based descriptors exhibit a permutation property that greatly reduces the equivocation of the eigenvalue-based axis labeling and moments-based polarity assignment in a computationally very efficient manner. In the present work, we show that this interesting permutation property is a consequence of the symmetry properties of regular polyhedra. Furthermore, we extend the invariance scheme to arbitrary 3D rotations by a discretization of the infinite space of $3 \mathrm{D}$ rotations followed by a nearest neighbor based approximate procedure employed to generate the necessary permutations.
\end{abstract}

Keywords: shape alignment; pose normalization; 3D shape descriptors; regular polyhedra; density-based framework

\section{INTRODUCTION}

For shape matching tasks, geometric transformations of 3D objects, such as translation, rotation, reflection, and isotropic rescaling (collectively denominated as similarity transformations) are often viewed as nuisance effects because they do not contribute to the semantic classification of a shape. While translation or rescaling problems are less problematic, there is still room for improvement in the alignment of objects with respect to rotations and reflections especially for 3D object retrieval $[1,2,3]$. Other applications that necessitate rigid-body alignment as a fundamental intermediate step include construction of new models by transferring parts from existing ones [4], transferring texture or surface detail [5], and hole filling [6], to name a few.

Principal component analysis (PCA) and its variants [2] constitute practically a universal tool for 3D pose normalization on a per object basis although they suffer from stability problems even in a semantically well-defined class [1, 2]. A particularly well known problem of PCA-based methods is the labeling of the principal axes. More specifically, the practice of axis labeling according to the decreasing rank of the eigenvalues of the object covariance matrix may lead to ambiguities especially when the eigenvalues are not well separated. A second related issue concerns the flipping invariance, that is, the assignment of the axis polarities [2]. In summary, the main problem is the fact that PCA-based axis alignment does not always yield consistent results due to variations in the extent of objects.

In our earlier work, we addressed the above issues within the density-based shape description framework (DBF) [1]. The pose normalization was made immune to the ambiguities of the PCA-based method by an exhaustive but systematic search over a restricted set of 3D rotations and reflections. In [1], we experimentally showed that density-based descriptors possess a permutation property that proves instrumental in efficiently reducing the equivocation of the eigenvalue-based axis labeling and moments-based polarity assignment. In the present work, first we show that this useful permutation property is a consequence of the symmetry properties of regular polyhedra. Furthermore, we extend the invariant alignment scheme to arbitrary $3 \mathrm{D}$ rotations. The extended algorithm is based on discretization of the infinite space of 3D rotations followed by a nearest neighbor approximate procedure that generates the necessary permutations. The outcomes are an exact and an approximate 3D shape alignment algorithm that are computationally efficient and constitute an alternative to PCA-based pose estimation.

The paper is organized as follow. In Section 2, we formulate the alignment problem as distance minimization between shape descriptors. In Section 3, we briefly review the density-based shape description framework putting more emphasis

Three-Dimensional Image Processing (3DIP) and Applications, edited by Atilla M. Baskurt,

Proc. of SPIE-IS\&T Electronic Imaging, SPIE Vol. 7526, 75260K · ( ) 2010 SPIE-IS\&T

CCC code: 0277-786X/10/\$18 - doi: 10.1117/12.838614 
on its aspects related to object alignment. In Section 4, after discussing the symmetry properties of regular polyhedra, we present exact and approximate versions of our alignment algorithm. In Section 5, we present our experimental results. In Section 6, we provide our concluding remarks.

\section{THE ALIGNMENT PROBLEM}

Given two centered and scale-matched $3 \mathrm{D}$ objects $A$ and $B$, we formulate the alignment problem as distance minimization over a certain finite set $\mathcal{G}$ of rigid-body transformations:

$$
\Gamma^{*}=\underset{\Gamma \in \mathcal{G}}{\arg \min } \operatorname{dist}(\Gamma[A], B)
$$

where the optimization is performed over a rigid-body transformation operator $\Gamma[\cdot]$, which can be a rotation, a reflection or a combination of both (a rotoreflection). First we need a mathematical representation for the 3D objects. If $A$ and $B$ are given in terms of 3D point or feature sets, the minimization problem is classically solved via a least-squares approach provided that correspondences between the entities in the two sets are given. However, automatically establishing such correspondences is a combinatorial problem, which requires the alignment problem be solved iteratively. Alternatively, one can try to find correspondences manually, which in turn is not practical for time-critical applications where several 3D objects must be automatically processed in batch mode. A workaround for this problem is to describe the objects by 3D shape descriptors that are defined on a common mathematical domain so that the minimization in (1) can be carried out in a correspondence-free manner. In this case, the problem in (1) is cast into:

$$
\Gamma^{*}=\underset{\Gamma \in \mathcal{G}}{\arg \min } \operatorname{dist}\left(\Gamma\left[\mathbf{f}_{A}\right], \mathbf{f}_{B}\right)
$$

where $\mathbf{f}_{A}$ and $\mathbf{f}_{B}$ are $K$-dimensional shape descriptor vectors of the objects $A$ and $B$, respectively. In (1) and (2), we employ the notation $\Gamma[\bullet]$ as a general operator that can be applied to $3 \mathrm{D}$ objects, to $3 \mathrm{D}$ points or features defined on these objects and also to their descriptors. This notational versatility simplifies the presentation of our analyses. Note that for an efficient implementation of (2), when the object $A$ undergoes a transformation $\Gamma$, we must be able to obtain the descriptor $\Gamma\left[\mathbf{f}_{A}\right]$ of its transformed version $\Gamma[A]$ by directly manipulating the entries of the original descriptor $\mathbf{f}_{A}$ recomputing anything from the original $3 \mathrm{D}$ object. Considering that $\mathcal{G}$ contains many transformations as will be exemplified in Section 4, this approach is obviously not practical. Accordingly, the chosen shape description methodology must allow a direct, efficient manipulation of descriptor entries under the 3D transformations $\Gamma$ from the set $\mathcal{G}$. Density-based shape descriptors satisfy this requirement as we explain in the next two sections.

\section{DENSITY-BASED SHAPE DESCRIPTORS}

\subsection{Overview}

For shape description, we employ the density-based framework (DBF). ). In [1] we have experimentally showed that $\mathrm{DBF}$ is a very effective shape description scheme for 3D retrieval applications. In DBF, the descriptor of a 3D object is derived from the probability density function (pdf) of multivariate local features computed on the object surface. Specifically, the samples of the estimated kernel density become the shape descriptor vector.

DBF enables the passage from local surface characterization to global shape description so that the aforementioned correspondence problem can be circumvented. In other words, DBF "registers" the local surface information obtained from different objects on a common grid where all descriptors are defined. In the context of rigid-body alignment, the surface feature should be pose-dependent, that is, when the object is rotated via a transformation $\Gamma$, the features should rotate as well. For instance, the radial and normal direction vectors at a surface point are such features taking their values on the unit sphere $\mathcal{S}^{2}$. Accordingly, for these features, the domain of the pdf is $\mathcal{S}^{2}$ and the pdf must be estimated on $3 \mathrm{D}$ points selected from $\mathcal{S}^{2}$. In density estimation terminology, such points are called targets. Judicious target selection plays a critical role in rigid alignment of objects, where alignment is realized by minimizing the distance between the density-based descriptors [1]. 


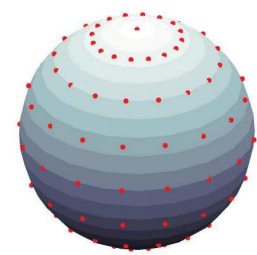

(a)

Spherical targets

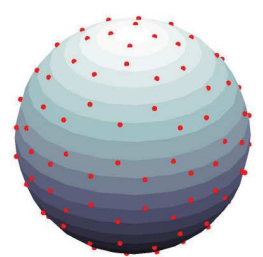

(b)

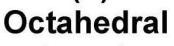
targets

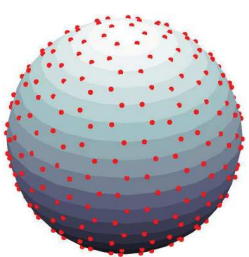

(c)

Icosahedral targets

Figure 1 (a) 128 target points obtained by uniformly sampling the domain $[0,2 \pi) \times[0, \pi)$ of the spherical parameters,

(b) 128 target points obtained by subdividing the octahedron twice, (c) 320 target points obtained by subdividing the icosahedron twice

\subsection{Target Selection for Pose-Dependent Features}

By pose-dependent features, we exclusively mean the radial direction or the normal direction vectors that change identically as the object subjected to a rigid-body transformation. The associated target selection problem is the one of sampling the unit sphere $\mathcal{S}^{2}$, for which several methods are available. An immediate option is to uniformly quantize the domain $[0,2 \pi) \times[0, \pi)$ of the spherical parameters. However, this quantization leads to an over-accumulation of points towards the poles of the unit-sphere; hence it produces a set of non-uniformly spaced target points (see Figure 1a). Since this produces biased samples for the density-based descriptor, this spherical sampling option is not desirable.

An alternative method to select targets from the unit sphere is based on recursively subdividing regular polyhedra, also known as Platonic solids, which consist of the tetrahedron (4 triangular faces), the cube (6 square faces) and its dual the octahedron ( 8 triangular faces), the dodecahedron (12 pentagonal faces) and its dual - the icosahedron (20 triangular faces). Note that regular polyhedra can be viewed as crude approximations to the sphere and consequently by successively subdividing a regular polyhedron, we can obtain finer approximations. The procedure to subdivide a regular polyhedron is quite simple. In octahedron subdivision for instance, we consider an octahedron circumscribed by the unit sphere, subdivide in four each of its eight triangular faces, and iterate likewise several times to obtain a subdivision as fine as we desire. After radially projecting the barycenters of the resulting triangles back to unit sphere, we obtain a set of target points that provides a more or less uniform partitioning of the unit sphere as shown in Figures 1b and 1c. Furthermore, this set of target points inherits the symmetry properties of the octahedron. It is in fact such symmetry properties that allow us to develop exact alignment algorithms as we explain in the next section.

\section{EXACT AND APPROXIMATE ALIGNMENTS}

\subsection{Symmetry Properties of Regular Polyhedra}

A regular polyhedron enjoys certain symmetry properties in the sense that it is possible to perform certain operations (certain rotations and reflections) which change the position of individual faces but leave the polyhedron in a position that is indistinguishable from its original position. In other words, the symmetrical nature of a polyhedron is characterized by the fact that it looks exactly the same from different viewpoints determined by its symmetry axes. Take the tetrahedron for instance (see first row in Table 1). The axis joining a vertex to the barycenter of the opposing face is such a symmetry axis that induces two different rotational symmetries. If one rotates the tetrahedron around this axis by $\pi / 3$ or $2 \pi / 3$ radians, the tetrahedron looks exactly the same. The only way to distinguish it from its former position is to first label the vertices (or the faces), and then to track these labels after the rotation. That is, the geometry of the tetrahedron is left intact; the only change is a permutation of the vertex labels, which is uniquely determined by the applied rotation. Since the tetrahedron has four vertices that can be paired with an opposing face, there are four symmetry axes of this type, each of which inducing two distinct rotations (by $\pi / 3$ and $2 \pi / 3$ radians), hence there are $4 \times 2=8$ rotations of this type in total. Another type of symmetry axis for the tetrahedron is the one joining the middle points of two opposing edges. This axis induces just a single rotational symmetry determined by a rotation by $\pi$ radians. 
Since there are three pairs of two opposing edges in the tetrahedron, the total number of rotations of this type is then six. These are the only symmetry axes for the tetrahedron. In general, denoting the number of symmetry axes of type $a$ by $N_{a}$ and the number of rotations induced by a specific symmetry axis of type $a$ by $n_{a}$ (including the identity rotation, $n_{a}$ is also called as the order of the associated axis), the total number $N$ of rotational symmetries of a regular polyhedron can be found by the following simple formula:

$$
N=\sum_{a \in \mathcal{A}} N_{a}\left(n_{a}-1\right)+1
$$

where the set $\mathcal{A}$ indexes the type of possible symmetry axes. Notice that, in (3) the identity symmetry for each axis is discounted in the summation, hence the term $n_{a}-1$; the +1 term outside the summation accounts for a single identity symmetry. To illustrate (3), let us reconsider the tetrahedron, which has two different types of symmetry axes, hence $|\mathcal{A}|=2$. There are four axes of type one (that is $N_{\mathrm{I}}=4$ ), each of order $n_{\mathrm{I}}=3$. There are three axes of type two (that is $N_{\text {II }}=3$ ), each of order $n_{\text {II }}=2$. Putting these in (3), we see that the total number of rotational symmetries of the tetrahedron is 12 . Note that these rotations are proper in the sense that the determinant of the corresponding operators is positive. To each such rotation, we can also associate a reflection (of negative determinant) to expand the set of all symmetries. This expanded set contains then $12 \times 2=24$ symmetries. Similar but a bit more involved analyses can be applied to the octahedron and the icosahedron as illustrated in Table 1. For our purposes in this work, the key fact that should be retained from these analyses is that the geometry of the polyhedron remains intact after any symmetry operation; the only change is a permutation of the vertex (or face) labels. Furthermore, this permutation is unique to the applied symmetry and it can be identified without any ambiguity.

Table 1 Regular Polyhedra and Their Symmetries ${ }^{1}$

\begin{tabular}{|c|c|c|c|c|}
\hline & Symmetry Axis I & Symmetry Axis II & Symmetry Axis III & No. of Symmetries \\
\hline $\begin{array}{c}\text { Tetrahedron } \\
4 \text { vertices } \\
4 \text { faces } \\
6 \text { edges }\end{array}$ & $\begin{array}{c}\text { Type: vertex-to-face } \\
N_{\mathrm{I}}=4 \\
n_{\mathrm{I}}=3\end{array}$ & $\begin{array}{c}\text { Type: edge-to-edge } \\
N_{\text {II }}=3 \\
n_{\text {II }}=2\end{array}$ & - No axis of Type III- & $\begin{array}{l}12 \text { rotations } \\
12 \text { rotoreflections } \\
24 \text { in total }\end{array}$ \\
\hline $\begin{array}{c}\text { Octahedron } \\
6 \text { vertices } \\
8 \text { faces } \\
12 \text { edges }\end{array}$ & $\begin{array}{c}\text { Type: vertex-to-vertex } \\
N_{\mathrm{I}}=3 \\
n_{\mathrm{I}}=4\end{array}$ & $\begin{array}{c}\text { Type: edge-to-edge } \\
N_{\text {II }}=6 \\
n_{\text {II }}=2\end{array}$ & $\begin{array}{c}\text { Type: face-to-face } \\
N_{\mathrm{III}}=4 \\
n_{\mathrm{III}}=3\end{array}$ & $\begin{array}{c}24 \text { rotations } \\
24 \text { rotoreflections } \\
48 \text { in total }\end{array}$ \\
\hline $\begin{array}{l}\text { Icosahedron } \\
12 \text { vertices } \\
20 \text { faces } \\
30 \text { edges }\end{array}$ & $\begin{array}{c}\text { Type: vertex-to-vertex } \\
\qquad \begin{array}{c}N_{\mathrm{I}}=6 \\
n_{\mathrm{I}}=5\end{array}\end{array}$ & $\begin{array}{c}\text { Type: edge-to-edge } \\
N_{\text {II }}=15 \\
n_{\text {II }}=2\end{array}$ & $\begin{array}{l}\text { Type: face-to-face } \\
\qquad \begin{array}{c}N_{\mathrm{III}}=10 \\
n_{\mathrm{III}}=3\end{array}\end{array}$ & $\begin{array}{c}60 \text { rotations } \\
60 \text { rotoreflections } \\
120 \text { in total }\end{array}$ \\
\hline
\end{tabular}

\footnotetext{
${ }^{1}$ The cube is the dual of the octahedron and the dodecahedron is the dual of the icosahedron. Accordingly, symmetries of the cube coincide with those of the octahedron and those of the dodecahedron with those of the icosahedron.
} 


\subsection{Exact Alignment}

Our formulation of the $3 \mathrm{D}$ object alignment requires the minimization of the distance between two descriptors over a finite set of rigid-body transformation (cf. Section 2). A procedure to perform this minimization is given in Table 2. This direct procedure exactly minimizes (2) when the 3D objects $A$ and $B$ differ by a transformation from the set $\mathcal{G}=\left\{\Gamma_{n}\right\}_{n=1}^{N}$. Computationally speaking, the critical step is step (1): $\mathbf{f} \leftarrow \Gamma_{n}\left[\mathbf{f}_{A}\right]$ where one evaluates the effect of the transformation $\Gamma_{n}$ on the descriptor $\mathbf{f}_{A}$. For arbitrary descriptors, this step necessitates that one first transform the object $A$ via $\Gamma_{n}[A]$ and then compute the descriptor $\mathbf{f}$ separately for each of the $N$ rigid-body transformations in the set $\mathcal{G}$. Obviously, this approach is computationally not practical.

The DBF scheme allows a viable implementation alternative since:

(i) The density-based descriptor corresponding to a pose-dependent feature (such as the local surface normal vector) consists of pdf values evaluated at target points selected on the unit sphere $\mathcal{S}^{2}$.

(ii) A regular polyhedron induces a set of symmetries each of which is associated with a specific rigid-body transformation operator. These symmetry transformations leave the polyhedron geometrically identical; they just induce permutations of the vertex (or face) labels. If the sampling points on the sphere are driven by the polyhedron, then obviously these points will also remain invariant under the symmetry transformations.

Table 2 Minimization procedure to align two 3D object models

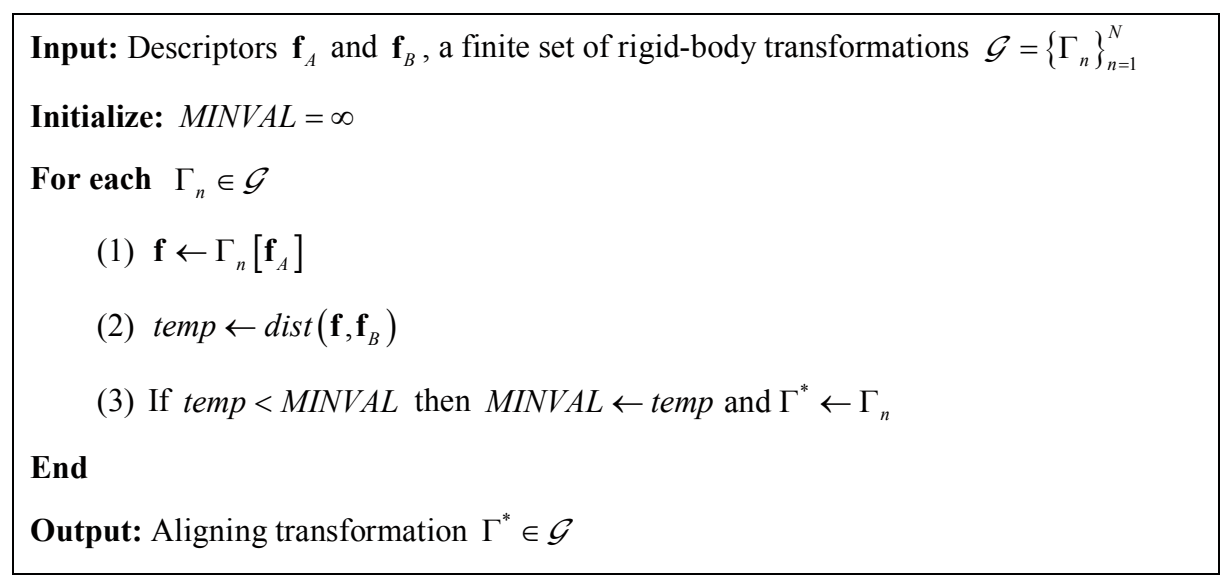

These two facts enable us to perform step (1): $\mathbf{f} \leftarrow \Gamma_{n}\left[\mathbf{f}_{A}\right]$ very efficiently provided that (i) the target points are selected by polyhedron subdivision, and (ii) the set of transformations $\mathcal{G}$ is restricted to coincide with the set of symmetries of the polyhedron used in selecting the targets. For instance, if the sampling points on the sphere are induced by an octahedron, since the latter has 48 symmetries (see Table 1) for any given $\Gamma_{n}$ among these, the computation of the descriptor $\mathbf{f} \leftarrow \Gamma_{n}\left[\mathbf{f}_{A}\right]$ for this transformation results from just a permutation of the entries in the descriptor $\mathbf{f}_{A}$, that is, $\mathbf{f} \leftarrow \pi_{n}\left[\mathbf{f}_{A}\right]$. This permutation can be performed very fast via simple swapping operations that can be read from a lookup table. In fact, the calculation of distances takes by far more time as compared to the $\mathbf{f} \leftarrow \pi_{n}\left[\mathbf{f}_{A}\right]$ mappings. 
Table 3 Nearest Neighbor Procedure for Generating Target Point Permutations Corresponding to an Arbitrary Set of 3D Rotations

Input: A finite set of arbitrary rotations $\mathcal{G}=\left\{\Gamma_{n}\right\}_{n=1}^{N}$, a finite set of target points $\mathcal{T}=\left\{t_{i}\right\}_{i=1}^{K} \subset \mathcal{S}^{2}$

For each $\Gamma_{n} \in \mathcal{G}$

For each $t_{i} \in \mathcal{T}$ find $\pi_{n}(i)=\underset{j}{\arg \min }\left\|t_{j}-\Gamma_{n}\left[t_{i}\right]\right\|_{2}$ End

End

Output: A set of permutations $\left\{\pi_{n}\right\}_{n=1}^{N}$ such that $\pi_{n} \leftrightarrow \Gamma_{n}, \forall n$.

\subsection{Approximate Alignment}

We have seen that as long as the rigid body transformations are limited to the symmetries of regular polyhedra exact alignment is possible. However, the only regular polyhedral are the ones listed in Table 1 [7] and they obviously represent a very small subset within the infinite set of 3D rotations. To tackle arbitrary 3D rotations then some sort of approximation is needed.

The approximate alignment technique relies on the discretization of the infinite space of $3 \mathrm{D}$ rotations, where the "discrete" transforms are given by $\mathcal{G}=\left\{\Gamma_{n}\right\}_{n=1}^{N}$ in Table 2. Notice that in general an arbitrary rotation will not induce a permutation of the target points (obtained by polyhedron subdivision) unless it belongs to a set of polyhedral symmetry. However, we can approximate permutations between target points (hence, descriptor vector entries) via a nearestneighbor procedure, as given in Table 3.

In Table 3, the notation $\pi_{n} \leftrightarrow \Gamma_{n}$ indicates that the permutation $\pi_{n}$ corresponds to some 3D rotation $\Gamma_{n}$ from the set $\mathcal{G}$. For an arbitrary rotation $\Gamma \notin \mathcal{G}$, the transformed target point $\Gamma\left(t_{i}\right)$ does not need to be exactly equal to one of the target points $t_{j} \in \mathcal{T}$ induced by a $\Gamma_{n}$ that belongs to the polyhedral symmetry set. As such, the permutations $\left\{\pi_{n}\right\}_{n=1}^{N}$ obtained using the nearest neighbor procedure using alignment procedure of Table 3, will correspond to approximate aligning transformations. We parameterize each rotation in $\mathcal{G}$ by the axis-angle parameterization defined as follows

$$
\Gamma=u u^{T}-\cos \theta\left(I-u u^{T}\right)+\sin \theta[u]_{\times}
$$

where $u \in \mathcal{S}^{2}$ is a unit-norm vector standing for the axis of rotation, $\theta \in[0,2 \pi)$ is the angle of rotation around that axis, $I$ is the $3 \times 3$ identity matrix and $[u]_{\times}$is the skew symmetric form of $u$. With this formula, we can represent any $3 \mathrm{D}$ rotation. In our experiments, we determine the rotation axes by selecting points from the unit sphere by polyhedron subdivision $^{2}$ and the rotation angles $\theta$ by uniformly quantizing the domain $[0,2 \pi)$. We adopt this discretization in order to sample the infinite space of $3 \mathrm{D}$ rotations as faithfully as possible.

\section{EXPERIMENTS}

\subsection{A Self-Alignment Test}

First, we show that our alignment algorithm can very accurately recover the pose of an object with respect to its original pose when the applied transformation coincides with a transformation from the predetermined set over which the distance minimization is carried out. In this experiment, the first set of poses coincides with the set of 48 octahedral symmetries, the second with the set of 120 icosahedral symmetries, and the third corresponds to a finite subset of

\footnotetext{
${ }^{2}$ This is not to be confused with target point selection. The procedure is the same but the purpose is different.
} 
arbitrary $3 \mathrm{D}$ rotations. This last set contains 512 rotations obtained using the axis-angle parameterization described in the previous section $^{3}$. To test our algorithm, we transformed a 3D human model using all the transformations in each set and checked whether the correct transformation was found. Accordingly, the number of test cases 48 for icosahedral symmetries, 120 for icosahedral symmetries, and 512 for the selected subset of arbitrary 3D rotations. We observed that for the sets of octahedral and icosahedral symmetries, the algorithm was able to find the correct pose in each case. For the subset of arbitrary 3D rotations, there were 14 cases (out of 512) where the recovered pose did not exactly correspond to the applied rotation. In other words, in $97.3 \%$ of the cases, the algorithm was successful. Note also that in the few cases where the recovered rotation was not correct, the estimated poses were nevertheless very close to the pose corresponding to the applied rotation (see instances in Figure 3). This self-alignment example illustrates the viability of our approach.

\section{Original \\ Pose}

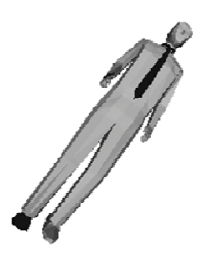
Recovered
Pose

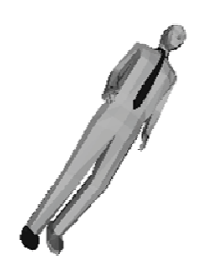

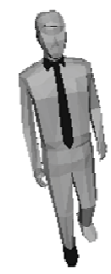

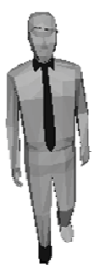

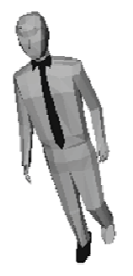
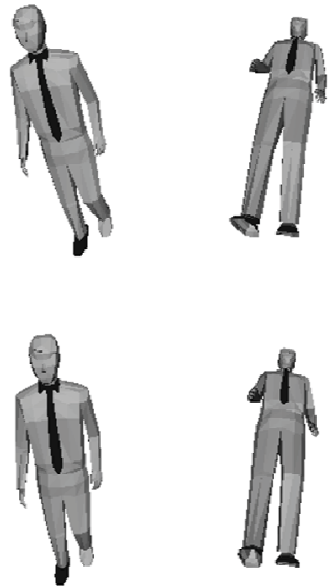
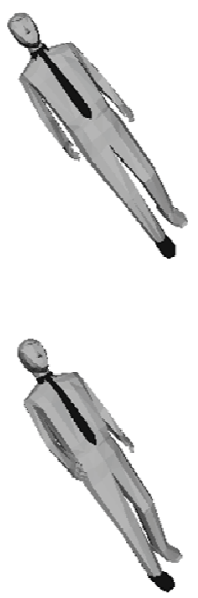

Figure 2 Illustration of erroneous pose recovery in the self-alignment experiments (see text)

\subsection{Alignment between Two Different Models of the Same Class}

In a second experiment, we evaluated the algorithm in aligning two different models of the same semantic class, when the pose differs by an arbitrary 3D rotation selected from the set of 512 arbitrary rotations considered in the previous experiment. We considered five shape classes (human, dog, plane, head, and wine glass) containing five different models each. Prior to the experiment, we manually aligned the models in each class. Then, for each of the 10 pairs of models in a given class, we held one model fixed and transformed the other using each of the 512 rotations. Accordingly, for a given class, there were $10 \times 512=5120$ alignment comparisons to make (i.e., $5 \times 5120=25600$ comparisons during the whole experiment). Excluding descriptor computation, the total time to carry out the alignments was about 25 minutes (the time of a single alignment is on the order of milliseconds thanks to the permutation property of the density-based descriptors). To quantify the performance, in addition to the number of strictly correct alignments, we also used an axis alignment measure defined as $\alpha=\left(1+\left\langle u^{*}, \hat{u}\right\rangle\right) / 2$, where $u^{*}$ is the axis of the applied rotation and $\hat{u}$ is the estimated axis of rotation. Observe that this measure is one when $u^{*}$ and $\hat{u}$ are in the same direction and polarity, and it is zero when they are antipodal. Table 4 provides performance statistics over 10 model pairings for each class. We observe that for human, dog, and plane shape classes, the algorithm satisfactorily aligns two models for the majority of applied rotations. The less compelling results for the head and wine glass classes can be attributed to the heterogeneity of the shape classes (and consequently to the variations in the shape descriptors), rather than to the alignment algorithm.

\footnotetext{
${ }^{3}$ First, we chose 64 rotation axes and then to each such axis we associated 8 distinct rotations by uniformly sampling the domain $[0,2 \pi)$, making 512 arbitrary $3 \mathrm{D}$ rotations in total.
} 
Table 4 Performance of the Algorithm in Aligning Objects of the Same Class

\begin{tabular}{|c|c|c|c|c|c|c|c|c|}
\hline & \multicolumn{3}{|c|}{ Percentage of Correct Alignments } & \multicolumn{4}{c|}{ Axis Alignment Measure $\alpha$} \\
\hline & Mean & Median & Min & Max & Mean & Median & Min & Max \\
\hline Human & 81.2 & 82.7 & 60.8 & 90.6 & 0.72 & 0.84 & 0.06 & 0.99 \\
\hline Dog & 71.9 & 89.8 & 21.3 & 96.7 & 0.89 & 0.98 & 0.30 & 0.99 \\
\hline Plane & 75.8 & 90.2 & 21.4 & 99.8 & 0.61 & 0.67 & 0.11 & 0.89 \\
\hline Head & 52.8 & 63.0 & 0.0 & 99.4 & 0.71 & 0.89 & 0.02 & 0.99 \\
\hline Wine glass & 45.9 & 51.9 & 13.8 & 65.3 & 0.66 & 0.72 & 0.02 & 1.00 \\
\hline
\end{tabular}

\section{CONCLUDING REMARKS}

In this work, we presented a computationally efficient correspondence-free rigid-body alignment algorithm for 3D objects. The problem of finding point (or feature) correspondences is circumvented by formulating the problem as distance minimization between shape descriptors. The computational efficiency of the algorithm is a consequence of the permutation property enjoyed by the chosen shape description methodology, the density-based framework. When the set of allowed transformations coincides with the set of symmetries of a regular polyhedron, this permutation property leads to exact alignment algorithms. We also presented an approximate version of the algorithm for arbitrary 3D rotations by relaxing the exact mapping requirement between descriptor target points using a nearest-neighbor based procedure. Our experiments showed that the algorithm can very accurately align two versions of the same object when they differ by a transformation selected from the set of allowed transformations. For the more difficult problem of aligning two different objects of the same classes, albeit not as accurate as for the self-alignment case, we obtained promising results (see Table 4). A fundamental limitation of the algorithm is that the solution set should be kept finite to allow a look-up table-based implementation. Although this procedure is computationally very efficient (a comparison takes around just one millisecond), it constitutes a limiting factor for $3 \mathrm{D}$ rotations that can be recovered. In the future, we plan to pursue more involved optimization procedures so that the algorithm can recover finer rotations.

\section{REFERENCES}

1. Akgul, C. B., Sankur, B., Yemez, Y., and Schmitt F., "3D Model Retrieval using Probability Density-Based Shape Descriptors ," IEEE Trans. on PAMI, vol. 31., no. 6, pp. 1117-1133, June 2009.

2. Vranic, D. V., 3D Model Retrieval, PhD Thesis, University of Leipzig, 2004.

3. Kazhdan, M., "An Approximate and Efficient Method for Optimal Rotation Alignment of 3D Models," IEEE Trans. on PAMI, vol. 29, no. 7, pp. 1221-1229, July 2007.

4. Yu, Y., Xu, D., Shi, X. , Bao, H., Guo, B., and Shum, H, "Mesh editing with Poisson-based gradient field manipulation," ACM Transactions on Graphics (SIGGRAPH 2004) 23, pp. 644-651, 2004.

5. Praun, E., Sweldens, W., and Schroeder, P., "Consistent mesh parameterizations," In Proceedings of the 28th Annual Conference on Computer Graphics and Interactive Techniques (SIGGRAPH 2001), 179-184, 2001.

6. Sharf, A., Alexa, M., and Cohen-Or, D, "Context-based surface completion", ACM Transactions on Graphics (SIGGRAPH 2004) 23, 878-887, 2004.

7. http://en.wikipedia.org/wiki/Platonic solid 